\title{
Interventions to improve adherence to cardiovascular disease guidelines: a systematic review
}

\author{
Rebecca A. Jeffery ${ }^{1 *}$, Matthew J. To ${ }^{1}$, Gabrielle Hayduk-Costa ${ }^{1}$, Adam Cameron³ ${ }^{3}$ Cameron Taylor ${ }^{2}$, \\ Colin Van Zoost ${ }^{1,3}$ and Jill A. Hayden ${ }^{4}$
}

\begin{abstract}
Background: Successful management of cardiovascular disease (CVD) is impaired by poor adherence to clinical practice guidelines. The objective of our review was to synthesize evidence about the effectiveness of interventions that target healthcare providers to improve adherence to CVD guidelines and patient outcomes.

Methods: We searched PubMed, EMBASE, Cochrane Library, PsycINFO, Web of Science and CINAHL databases from inception to June 2014, using search terms related to adherence and clinical practice guidelines. Studies were limited to randomized controlled trials testing an intervention to improve adherence to guidelines that measured both a patient and adherence outcome. Descriptive summary tables were created from data extractions. Meta-analyses were conducted on clinically homogeneous comparisons, and sensitivity analyses and subgroup analyses were carried out where possible. GRADE summary of findings tables were created for each comparison and outcome.
\end{abstract}

Results and Discussion: We included 38 RCTs in our review. Interventions included guideline dissemination, education, audit and feedback, and academic detailing. Meta-analyses were conducted for several outcomes by intervention type. Many comparisons favoured the intervention, though only the adherence outcome for the education intervention showed statistically significant improvement compared to usual care (standardized mean difference $=0.58$ [95 \% confidence interval 0.35 to 0.8$]$ ]).

Conclusions: Many interventions show promise to improve practitioner adherence to CVD guidelines. The quality of evidence and number of trials limited our ability to draw conclusions.

Keywords: Clinical practice guidelines, Cardiovascular disease, Adherence, Systematic review

\section{Background}

Cardiovascular disease (CVD) is a leading cause of death in Canada [1]. Successful management of CVD involves not only the treatment of a specific disease, but also treating and preventing risk factors for CVD, including diabetes, dyslipidemia and hypertension [1-3]. However, the management of CVD is complicated by the large number of clinical practice guidelines available for conditions that contribute to this disease. An article by Ray et al. noted there are also discrepancies in recommendations across guidelines, potentially contributing to low adherence rates

\footnotetext{
* Correspondence: rjeffer@dal.caAQ3

${ }^{1}$ Faculty of Medicine, Dalhousie University, Mailbox 354, 5849 University Avenue, Halifax, NS, CanadaB3H 4R2

Full list of author information is available at the end of the article
}

$[2,4,5]$. A harmonized guideline by Tobe et al. (2011) found there are over 400 recommendations for managing risk factors for heart disease [3].

Given the complexity of the management of this illness, it is imperative that practitioners use guidelines, and the most appropriate guidelines, in caring for patients with CVD and risk factors for CVD. The impact of guideline implementation has been illustrated previously; a review by Grimshaw and Russell found that using guidelines improved clinical practice [6]. Despite evidence to support the use of guidelines, there remains a gap in their implementation [7].

The dissemination of guidelines alone has little to no effect on practice [8], thus many studies have investigated interventions of varying intensity to increase the 
uptake of clinical practice guidelines. Numerous studies of interventions to improve the uptake of guidelines in CVD prevention are available. However, their overall impact on guideline adherence and clinical outcomes is unclear. Unverzagt et al. [9] published a systematic review on a similar topic that focused on primary care physicians' adherence to guidelines, wherein they demonstrated these interventions can have an impact on adherence outcomes. It is important to determine the effect of these interventions on other healthcare providers, as well as determine the impact of these interventions on clinical outcomes, which is yet to be addressed in the literature to our knowledge.

We identified and synthesized the available research evidence about the effectiveness of interventions that target healthcare providers to improve adherence to CVD prevention and treatment guidelines and clinical outcomes. Our secondary objective was to explore characteristics of guideline implementation interventions and contexts that are associated with increased effectiveness. This leads to our research question: what is the most effective intervention to improve the implementation of, uptake of, or adherence to cardiovascular disease-related clinical practice guidelines by healthcare providers in randomized controlled trials?

\section{Methods}

As this research did not involve the collection of primary data, we did not seek ethics approval. This review has been registered with PROSPERO 2014:CRD42014010111. Available from http://www.crd.york.ac.uk/PROSPERO/ display_record.asp?ID=CRD42014010111

\section{Search}

A systematic search was conducted using search terms related to "adherence" and "clinical practice guidelines", which was refined with the help of a medical librarian. We searched the following databases: PubMed, EMBASE, Cochrane Library (including CENTRAL, DARE and HTAs), PsycINFO, Web of Science and CINAHL (all available years, up to June 2014). Grey literature was also searched, including clinicaltrials.gov to identify potential new studies, ICTRP registry database, and ProQuest thesis database. Our search strategy did not impose any limits on language of publication (Additional file 1).

\section{Inclusion criteria \\ Study design}

The included studies were limited to randomized controlled trials (RCTs). We included all types of RCTs, including cluster RCTs, and nested designs.

\section{Population}

Studies that enrolled any registered healthcare providers were included. Subgroups of interest for our analyses included comparing physician participants to other healthcare providers (non-physicians). We excluded trials if less than $75 \%$ of the participants included were certified, regulated healthcare providers.

\section{Intervention}

All studies that evaluated the impact of an intervention on the implementation of, uptake of, or adherence to a clinical practice guideline by a health care provider were included. The guideline of concern had to relate to the prevention or management of CVD, including risk factor management for any of: diabetes, dyslipidemia or hypertension. Guideline definitions were based on authors stating a guideline to be such. A study was deemed to be about the implementation or adherence to a guideline if the trial report explicitly stated that improving use of a clinical practice guideline was the focus of the intervention. Types of interventions included: academic detailing, audit and feedback, educational sessions, continuing medical education (CME) sessions, and 'other' (such as reminders or decision support systems).

\section{Comparison group}

We selected studies that included at least one control group. Comparison groups included usual care, a similar guideline implementation intervention of differing intensity or duration than the main intervention group, or no intervention (receipt of the intervention at a different time than the intervention group, such as after data collection).

\section{Primary outcomes}

We included trials that reported both a measure of guideline adherence and at least one clinical outcome. Measures of adherence included self-reported adherence, prescription review, and chart review. We included studies reporting any relevant clinical outcomes and considered the following groups of outcomes for analyses: mortality, hospitalizations, quality of life, and disease targets. Outcomes assessed at similar time points were combined in our analysis as short term (3-6 months), and long term (7 months or longer).

\section{Study selection and data extraction}

Articles were screened based on title and abstract using the inclusion criteria, then based on full text by two independent reviewers. Discrepancies were resolved by consensus.

Data from included articles was extracted in duplicate by independent extractors. We extracted study characteristics (study design, setting and population), a description 
of the intervention (the type of intervention, providers, and resources involved), comparison intervention, risk of bias, outcome measurement and results, and funding for the study. Risk of bias was assessed using the Cochrane Risk of Bias tool for RCTs [10]. All discrepancies between extractors were resolved through consensus. Data was managed using spreadsheets created for each extractor. Authors were contacted after data extraction and consensus meetings were completed to request missing data and to check the accuracy of our extractions.

\section{Data analysis}

We conducted descriptive analyses of included studies. We conducted meta-analyses (MA) for outcome results when there was sufficient clinical homogeneity across the studies. Clinical homogeneity was based on similar study characteristics (intervention type, outcome and follow-up point of interest). Meta-analyses were conducted in Review Manager (RevMan 5), using a random effects model and forest plots were generated. Intraclass correlation coefficient (ICC) for cluster RCTs were used in our meta-analyses to calculate the effective sample size to ensure the effect of clustering was taken into account in our analyses, as per the Cochrane Handbook [11]. A Z-test was used to assess statistical significance of meta-analysis results and a $p<0.05$ was considered significant.

The adherence and patient outcomes were measured as both dichotomous and continuous outcome measures. Odds ratios (OR) and $95 \%$ confidence intervals (CI) were calculated for use in the MA for dichotomous outcomes. Standardized mean differences (SMD) were used for continuous outcomes, as outcomes measuring the same construct were measured on different scales. Most continuous outcome analyses looked at the differences in mean change of each group from baseline, and this value was used in the MA, though some trials reported follow up results in each group, wherein we calculated the change score for each group to use in our MA. In order to impute the standard deviation for the change score in this instance, the standard deviation of the change score from another similar study was used.

We conducted sensitivity analyses to determine the robustness of our results, comparing the results of our analyses including and excluding studies with imputed standard deviations, and excluding studies with high risk of bias (greater than 3 domains rated as high risk of bias). We conducted subgroup analyses considering participant subgroups (physician participants and other healthcare providers), and considering the condition that was the focus of the guideline in the study (acute and chronic CVD conditions or risk factors). We planned to create funnel plots to investigate potential publication bias if at least ten studies were included for a given outcome, however this was not possible. We present a summary of the overall strength of evidence available using GRADE Summary of Findings tables produced using GRADEpro.

\section{Results}

\section{Results of the search}

We identified 12,255 potentially relevant unique citations. We excluded 12,033 citations during the initial abstract and title screening. We reviewed 222 full text publications and included 38 studies in the review [12-54] (Fig. 1).

\section{Included studies (Table 1)}

Eighteen studies took place in the USA, 14 were completed in Europe (the Netherlands, Italy, England, and Norway), two took place in Canada, one in South Africa, one in Brazil, one in Asia-Pacific area, and one in the Virgin Islands. Thirty-five studies included an intervention to improve physician use of guidelines and ten of those studies included a nurse as a target for the intervention; two studies focused on nurses alone, and one study focused on pharmacists. The most common intervention type was educational focused intervention (18/38), followed by audit and feedback (9/38), academic detailing focused interventions $(4 / 38)$, comprehensive interventions that included education, audit and feedback and an academic detailing component (2/38), and "other" interventions that did not fall into any pre-designated category $(8 / 38)$. Seven trials included more than one intervention group. All studies included an adherence outcome, as per our inclusion criterion. Disease target was the most common clinical outcome reported (33/38 trials), followed by mortality (11/38). Hospitalization and quality of life data were also reported in $8 / 38$ and $6 / 38$ trials, respectively.

\section{Risk of bias in included studies}

Risk of bias summary graphs and tables were created using RevMan (Figs. 2 and 3). Risk of bias was often assessed as unclear due to poor reporting of a methodological procedure. The majority of trials (33/38) were cluster RCTs, therefore additional risk of bias criteria were included for these studies. Random sequence generation was most often assessed to be low risk of bias, while blinding of participants was most commonly rated as high risk of bias.

\section{Effects of interventions}

\section{Education intervention}

Seventeen trials tested an education-focused intervention and were included in a meta-analysis. These trials overall favoured the intervention, and one meta-analysis was statistically significant. Seven trials (2545 subjects) reported mortality outcomes, three of which reported mortality at a short term time point with an overall odds 


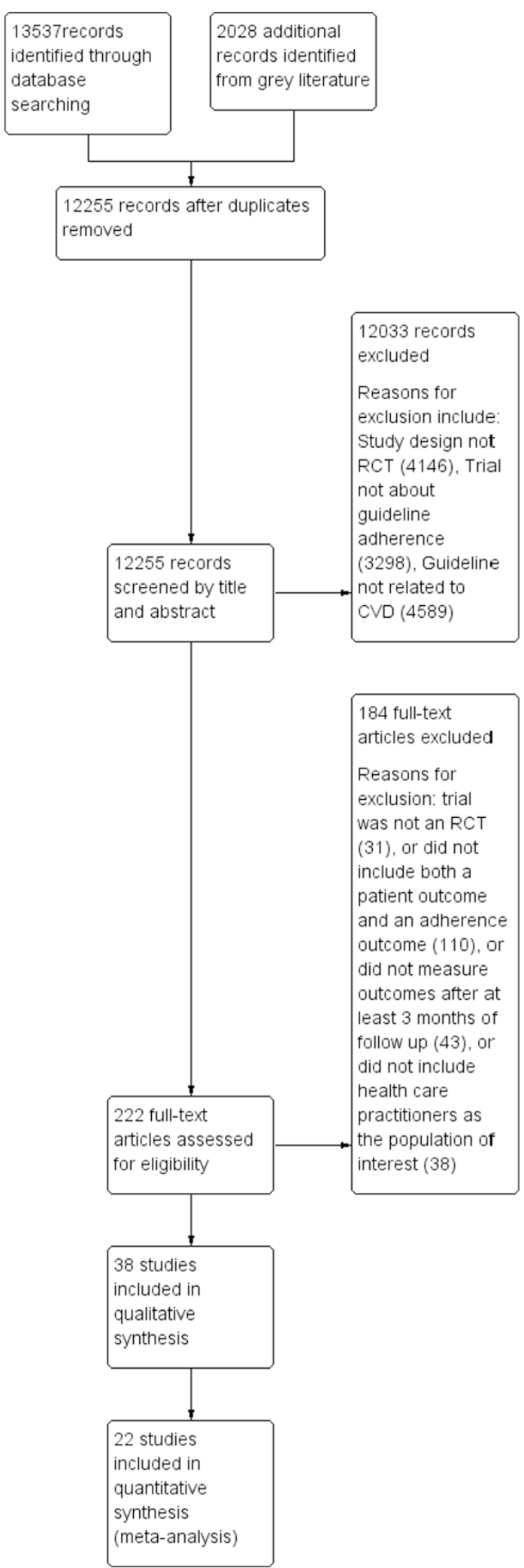

Fig. 1 PRISMA flow chart of study inclusions ratio of 0.54 ( $95 \%$ CI 0.2 to 1.42). Four trials reported mortality at a long term time point with an overall odds ratio of 0.48 (95\% CI 0.11 to 1.98). Four trials (979 subjects) reported hospitalizations as an outcome at a long term time point. The overall odds ratio for this outcome was 0.88 (95\% CI 0.54 to 1.41 ). Six trials (2145 subjects) reported disease target results at a short term time point $(\mathrm{SMD}=-0.32(95 \% \mathrm{CI}-0.71$ to 0.07$))$ and five trials (2732 subjects) reported this outcome at a long term time point $(\mathrm{SMD}=-0.09(95 \% \mathrm{CI}-0.24$ to 0.07$))$ (Fig. 5). Seventeen trials reported adherence outcome data, six (2306 subjects) reported dichotomous data at a short term time point $(\mathrm{OR}=2.11$ (95\% CI 0.90 to 4.97$)$ ), four trials (322 subjects) reported continuous data at a short term time point (Fig. 4) and eight trials (6019 subjects) reported dichotomous data at a long term time point $(\mathrm{OR}=1.05$ (95\% CI 0.82 to 1.34$)$ ) (Fig. 6).

\section{Audit and feedback}

Nine trials included an intervention that focused on audit and feedback, and seven of those trials reported data sufficient to be included in meta-analyses. Three trials (2240 subjects) reported disease target results at a long term time point with an overall effect of -0.44 SMD (95 \% CI -1.05 to 0.17) (Fig. 5). Six trials (2983 subjects) reported adherence data at a long term time point with an overall odds ratio of 1.39 (95\% CI 0.88 to 2.21) (Fig. 6).

\section{Academic detailing}

Four trials (6017 subjects) included academic detailing as the focus of the intervention and all of these trials reported data that was included in a meta-analysis for adherence outcome. The overall odds ratio for this comparison was 1.32 (95\% CI 0.88 to 1.96) (Fig. 6).

\section{Other interventions}

Eight trials included an intervention whose focus did not fit these previous groups. Four trials (1782 subjects) included a decision support tool as the focus of their intervention. The overall odds ratio for this comparison was 1.19 (95\% CI 0.83 to 1.70) (Fig. 6).

\section{Sensitivity and subgroup analyses}

Sensitivity analysis investigating the impact of imputed standard deviations in continuous data was possible in the education intervention outcome for the disease target outcome at a short term time point. The pooled SMD from six studies in this comparison was -0.32 (95\% CI -0.71 to 0.07 ), while the estimate from the sensitivity analysis, with studies that included imputed standard deviation removed, was -0.27 ( $95 \% \mathrm{CI}-0.71$ to 0.17 ). Another sensitivity analysis, investigating the impact of high 
Table 1 Characteristics of included studies

\begin{tabular}{|c|c|c|c|c|c|c|c|c|c|c|}
\hline Study ID & Topic of trial & $\begin{array}{l}\text { Study } \\
\text { Design }\end{array}$ & $\begin{array}{l}\text { Population } \\
\text { description }\end{array}$ & Setting & $\begin{array}{l}\text { Intervention Description; } \\
\text { Intervention } 2 \text { description (if } \\
\text { applicable) }\end{array}$ & Type & $\begin{array}{l}\text { Duration } \\
\text { of } \\
\text { treatment } \\
\text { period }\end{array}$ & $\begin{array}{l}\text { Comparison } \\
\text { intervention }\end{array}$ & Outcomes measured & $\begin{array}{l}\text { Risk of } \\
\text { bias } \\
\text { rating }\end{array}$ \\
\hline $\begin{array}{l}\text { Ansari, } \\
2003\end{array}$ & $\begin{array}{l}\text { Use of beta-blockers in } \\
\text { congestive heart } \\
\text { failure }\end{array}$ & CRCT & $\begin{array}{l}\text { Specialist doctors and } \\
\text { nurse practitioners, } \\
\text { patients with CHF }\end{array}$ & $\begin{array}{l}\text { USA, urban } \\
\text { medical } \\
\text { centre }\end{array}$ & $\begin{array}{l}\text { Nurse facilitator plus } \\
\text { healthcare provider } \\
\text { educational sessions; provider } \\
\text { and patient reminder letters }\end{array}$ & $\begin{array}{l}\text { Other type: } \\
\text { Nurse } \\
\text { facilitator; } \\
\text { notifications }\end{array}$ & 1 year & $\begin{array}{l}\text { Educational } \\
\text { sessions, no } \\
\text { nurse facilitator }\end{array}$ & $\begin{array}{l}\text { Mortality, } \\
\text { hospitalization, } \\
\text { adherence (prescription } \\
\text { review, chart review) }\end{array}$ & $\begin{array}{l}\text { High } \\
\text { risk of } \\
\text { bias }\end{array}$ \\
\hline Baker, 2003 & $\begin{array}{l}\text { Guidelines in } \\
\text { prioritised review } \\
\text { criteria }\end{array}$ & CRCT & $\begin{array}{l}\text { Family doctors, } \\
\text { patients with angina }\end{array}$ & $\begin{array}{l}\text { England, } \\
\text { general } \\
\text { practices }\end{array}$ & $\begin{array}{l}\text { Review criteria; criteria plus } \\
\text { feedback }\end{array}$ & $\begin{array}{l}\text { Other type: } \\
\text { review criteria }\end{array}$ & 12 months & $\begin{array}{l}\text { Guideline } \\
\text { dissemination } \\
\text { alone }\end{array}$ & $\begin{array}{l}\text { Disease target } \\
\text { (cholesterol), adherence } \\
\text { (prescription review, } \\
\text { chart review) }\end{array}$ & $\begin{array}{l}\text { Low } \\
\text { risk of } \\
\text { bias }\end{array}$ \\
\hline $\begin{array}{l}\text { Bertoni, } \\
2009\end{array}$ & $\begin{array}{l}\text { Physician adherence } \\
\text { to ATP III guidelines }\end{array}$ & CRCT & Family doctors & $\begin{array}{l}\text { USA, primary } \\
\text { care practices }\end{array}$ & $\begin{array}{l}\text { CDSS, educational sessions, } \\
\text { academic detailing, CME } \\
\text { sessions }\end{array}$ & $\begin{array}{l}\text { Education }+ \\
\text { audit and } \\
\text { feedback }+ \\
\text { academic } \\
\text { detailing + CME } \\
\text { session }\end{array}$ & 2 years & $\begin{array}{l}\text { educational } \\
\text { sessions, CME } \\
\text { sessions, } \\
\text { guideline } \\
\text { mailed to } \\
\text { participants }\end{array}$ & $\begin{array}{l}\text { Disease target } \\
\text { (cholesterol), adherence } \\
\text { (prescription review, } \\
\text { chart review) }\end{array}$ & $\begin{array}{l}\text { High } \\
\text { risk of } \\
\text { bias }\end{array}$ \\
\hline $\begin{array}{l}\text { Berwanger, } \\
2012\end{array}$ & $\begin{array}{l}\text { Multifaceted quality } \\
\text { improvement } \\
\text { intervention in ACS } \\
\text { patients }\end{array}$ & CRCT & $\begin{array}{l}\text { Patients with ACS at } \\
\text { general public } \\
\text { hospitals }\end{array}$ & $\begin{array}{l}\text { Brazil, public } \\
\text { hospitals }\end{array}$ & $\begin{array}{l}\text { Training, reminders, checklists, } \\
\text { case management, } \\
\text { educational sessions }\end{array}$ & Education & 8 months & Routine care & $\begin{array}{l}\text { Mortality, major adverse } \\
\text { cardiac events, } \\
\text { adherence (prescription } \\
\text { review) }\end{array}$ & $\begin{array}{l}\text { Low } \\
\text { risk of } \\
\text { bias }\end{array}$ \\
\hline $\begin{array}{l}\text { Bonds, } \\
2009\end{array}$ & $\begin{array}{l}\text { Compliance to JNC } 7 \\
\text { guidelines to improve } \\
\text { blood pressure }\end{array}$ & CRCT & Family doctors & $\begin{array}{l}\text { USA, primary } \\
\text { care practices }\end{array}$ & $\begin{array}{l}\text { Educational sessions, } \\
\text { dissemination of guidelines, } \\
\text { academic detailing for } \\
\text { physicians, feedback on blood } \\
\text { pressure control }\end{array}$ & $\begin{array}{l}\text { Education }+ \\
\text { audit and } \\
\text { feedback }+ \\
\text { academic } \\
\text { detailing + CME } \\
\text { sessions }\end{array}$ & 2 years & $\begin{array}{l}\text { Similar to } \\
\text { intervention } \\
\text { but focused on } \\
\text { ATPIII } \\
\text { guidelines }\end{array}$ & $\begin{array}{l}\text { Disease target (BP), } \\
\text { adherence (prescription } \\
\text { review, chart review) }\end{array}$ & $\begin{array}{l}\text { Low } \\
\text { risk of } \\
\text { bias }\end{array}$ \\
\hline $\begin{array}{l}\text { Browner, } \\
1994\end{array}$ & $\begin{array}{l}\text { CME and follow up to } \\
\text { improve detection and } \\
\text { treatment of high } \\
\text { cholesterol }\end{array}$ & CRCT & $\begin{array}{l}\text { Family and internal } \\
\text { medicine doctors }\end{array}$ & $\begin{array}{l}\text { USA, general } \\
\text { practices }\end{array}$ & $\begin{array}{l}\text { CME seminar; Intensive CME } \\
\text { (office visits and educational } \\
\text { materials) }\end{array}$ & $\begin{array}{l}\text { Education }+ \\
\text { CME sessions }\end{array}$ & 18 months & $\begin{array}{l}\text { Educational } \\
\text { sessions }\end{array}$ & $\begin{array}{l}\text { Disease target } \\
\text { (cholesterol), adherence } \\
\text { (chart review) }\end{array}$ & $\begin{array}{l}\text { High } \\
\text { risk of } \\
\text { bias }\end{array}$ \\
\hline $\begin{array}{l}\text { Carter, } \\
2009\end{array}$ & $\begin{array}{l}\text { Physician and } \\
\text { pharmacist } \\
\text { collaborative model to } \\
\text { improve blood } \\
\text { pressure }\end{array}$ & CRCT & $\begin{array}{l}\text { Family doctors, } \\
\text { patients with } \\
\text { hypertension }\end{array}$ & $\begin{array}{l}\text { USA, } \\
\text { community } \\
\text { based family } \\
\text { medicine }\end{array}$ & $\begin{array}{l}\text { Collaborative model, team } \\
\text { building exercises, training } \\
\text { sessions, educational sessions }\end{array}$ & $\begin{array}{l}\text { Education + } \\
\text { other } \\
\text { (collaborative } \\
\text { model) }\end{array}$ & 6 months & $\begin{array}{l}\text { Collaborative } \\
\text { model }\end{array}$ & $\begin{array}{l}\text { Disease target (BP), } \\
\text { guideline adherence } \\
\text { tool }\end{array}$ & $\begin{array}{l}\text { High } \\
\text { risk of } \\
\text { bias }\end{array}$ \\
\hline $\begin{array}{l}\text { De } \\
\text { Lusignan, } \\
2013\end{array}$ & $\begin{array}{l}\text { Audit based education } \\
\text { to reduce blood } \\
\text { pressure }\end{array}$ & CRCT & $\begin{array}{l}\text { Mixed health care } \\
\text { professionals }\end{array}$ & $\begin{array}{l}\text { United } \\
\text { Kingdom, } \\
\text { primary care }\end{array}$ & $\begin{array}{l}\text { Audit based education } \\
\text { consisting of workshops; } \\
\text { academic detailing plus } \\
\text { workshops }\end{array}$ & $\begin{array}{l}\text { Education + } \\
\text { audit and } \\
\text { feedback; } \\
\text { academic } \\
\text { detailing }\end{array}$ & 2 years & Usual care & $\begin{array}{l}\text { Mortality, major adverse } \\
\text { cardiac events, disease } \\
\text { target (BP), adherence } \\
\text { (prescription review) }\end{array}$ & $\begin{array}{l}\text { Low } \\
\text { risk of } \\
\text { bias }\end{array}$ \\
\hline \multirow[t]{2}{*}{$\begin{array}{l}\text { Deales, } \\
2014\end{array}$} & $\begin{array}{l}\text { Team based approach } \\
\text { to disease and care } \\
\text { management }\end{array}$ & cRCT & $\begin{array}{l}\text { Mixed health care } \\
\text { professionals }\end{array}$ & $\begin{array}{l}\text { Italy, primary } \\
\text { care groups }\end{array}$ & $\begin{array}{l}\text { Recommendations as } \\
\text { textbooks and decision } \\
\text { algorithms, education sessions }\end{array}$ & Education & 12 months & Usual care & $\begin{array}{l}\text { Disease target (HbA1c), } \\
\text { adherence (chart } \\
\text { review) }\end{array}$ & $\begin{array}{l}\text { High } \\
\text { risk of } \\
\text { bias }\end{array}$ \\
\hline & & CRCT & T1D and T2D patients & & & & 1 year & Usual care & & \\
\hline
\end{tabular}


Table 1 Characteristics of included studies (Continued)

\begin{tabular}{|c|c|c|c|c|c|c|c|c|c|c|}
\hline $\begin{array}{l}\text { Dijkstra, } \\
2006\end{array}$ & $\begin{array}{l}\text { Implementation } \\
\text { strategies for diabetes } \\
\text { guidelines }\end{array}$ & & & $\begin{array}{l}\text { The } \\
\text { Netherlands, } \\
\text { hospitals }\end{array}$ & $\begin{array}{l}\text { Educational meetings, } \\
\text { feedback, reminder card; } \\
\text { diabetes passport, education }\end{array}$ & $\begin{array}{l}\text { Education }+ \\
\text { audit and } \\
\text { feedback }\end{array}$ & & & $\begin{array}{l}\text { Disease target (HbA1c), } \\
\text { adherence (chart } \\
\text { review) }\end{array}$ & $\begin{array}{l}\text { High } \\
\text { risk of } \\
\text { bias }\end{array}$ \\
\hline Eaton, 2011 & $\begin{array}{l}\text { Multimodal } \\
\text { intervention to } \\
\text { improve screening } \\
\text { and management of } \\
\text { hyperlipidemic } \\
\text { patients }\end{array}$ & $\mathrm{CRCT}$ & Family doctors & $\begin{array}{l}\text { USA, primary } \\
\text { care practices }\end{array}$ & $\begin{array}{l}\text { PDA with decision support } \\
\text { and education toolkit and } \\
\text { academic detailing }\end{array}$ & $\begin{array}{l}\text { Academic } \\
\text { detailing }\end{array}$ & 12 months & $\begin{array}{l}\text { PDA with } \\
\text { decision } \\
\text { support but } \\
\text { minimal follow } \\
\text { up }\end{array}$ & $\begin{array}{l}\text { Disease target } \\
\text { (cholesterol), adherence } \\
\text { (chart review) }\end{array}$ & $\begin{array}{l}\text { Low } \\
\text { risk of } \\
\text { bias }\end{array}$ \\
\hline $\begin{array}{l}\text { Eccles, } \\
2002\end{array}$ & $\begin{array}{l}\text { Computerised decision } \\
\text { support system to } \\
\text { implement angina } \\
\text { guidelines }\end{array}$ & $\mathrm{CRCT}$ & Family doctors & $\begin{array}{l}\text { England, } \\
\text { general } \\
\text { practices }\end{array}$ & $\begin{array}{l}\text { Computer decision support } \\
\text { that provided access to } \\
\text { guidelines }\end{array}$ & Other: CDSS & 12 months & $\begin{array}{l}\text { Same } \\
\text { intervention } \\
\text { but asthma } \\
\text { guideline } \\
\text { provided }\end{array}$ & $\begin{array}{l}\text { Quality of life, } \\
\text { adherence (chart } \\
\text { review) }\end{array}$ & $\begin{array}{l}\text { Low } \\
\text { risk of } \\
\text { bias }\end{array}$ \\
\hline $\begin{array}{l}\text { Feldman, } \\
2009\end{array}$ & $\begin{array}{l}\text { Simplified algorithm } \\
\text { for treatment of } \\
\text { hypertension }\end{array}$ & $\mathrm{CRCT}$ & $\begin{array}{l}\text { Family practices, } \\
\text { patients with } \\
\text { hypertension }\end{array}$ & $\begin{array}{l}\text { Canada, } \\
\text { family } \\
\text { practices }\end{array}$ & $\begin{array}{l}\text { Algorithm, aids, one follow up } \\
\text { meeting, educational materials } \\
\text { and sessions }\end{array}$ & $\begin{array}{l}\text { Education + } \\
\text { Other } \\
\text { (algorithms) }\end{array}$ & 6 months & $\begin{array}{l}\text { Educational } \\
\text { sessions and } \\
\text { guidelines }\end{array}$ & $\begin{array}{l}\text { Mortality, disease target } \\
(\mathrm{BP}), \text { adherence (chart } \\
\text { review) }\end{array}$ & $\begin{array}{l}\text { Low } \\
\text { risk of } \\
\text { bias }\end{array}$ \\
\hline Fihn, 2011 & $\begin{array}{l}\text { Collaborative care } \\
\text { model based } \\
\text { intervention to } \\
\text { improve angina } \\
\text { management }\end{array}$ & $\mathrm{CRCT}$ & $\begin{array}{l}\text { Family doctors, } \\
\text { patients with angina }\end{array}$ & $\begin{array}{l}\text { USA, } \\
\text { academic } \\
\text { primary care } \\
\text { clinics }\end{array}$ & $\begin{array}{l}\text { Expert advice, progress } \\
\text { evaluations, education }\end{array}$ & Education & 12 months & Usual care & $\begin{array}{l}\text { Mortality, disease target, } \\
\text { adherence (chart } \\
\text { review) }\end{array}$ & $\begin{array}{l}\text { Low } \\
\text { risk of } \\
\text { bias }\end{array}$ \\
\hline $\begin{array}{l}\text { Fretheim, } \\
2006\end{array}$ & $\begin{array}{l}\text { Tailored intervention } \\
\text { to support } \\
\text { implementation of } \\
\text { CVD guidelines }\end{array}$ & $\mathrm{CRCT}$ & $\begin{array}{l}\text { Family practices, } \\
\text { hypertensive or } \\
\text { hypercholesterolemic } \\
\text { patients }\end{array}$ & $\begin{array}{l}\text { Norway, } \\
\text { general } \\
\text { practices }\end{array}$ & $\begin{array}{l}\text { Tailored intervention including } \\
\text { reminders, audit and feedback } \\
\text { and education }\end{array}$ & $\begin{array}{l}\text { Education + } \\
\text { audit and } \\
\text { feedback }\end{array}$ & 12 months & $\begin{array}{l}\text { Passive } \\
\text { dissemination }\end{array}$ & $\begin{array}{l}\text { Disease target } \\
\text { (cholesterol, BP), } \\
\text { adherence (prescription } \\
\text { review, chart review) }\end{array}$ & $\begin{array}{l}\text { Low } \\
\text { risk of } \\
\text { bias }\end{array}$ \\
\hline Gill, 2009 & $\begin{array}{l}\text { EMR-based } \\
\text { intervention for lipid } \\
\text { management }\end{array}$ & CRCT & $\begin{array}{l}\text { Family doctors, } \\
\text { general internists }\end{array}$ & $\begin{array}{l}\text { USA, } \\
\text { academic } \\
\text { family } \\
\text { practice }\end{array}$ & $\begin{array}{l}\text { EMR disease management } \\
\text { tool }\end{array}$ & $\begin{array}{l}\text { Other } \\
\text { (integration } \\
\text { into EMR) }\end{array}$ & 12 months & Usual care & $\begin{array}{l}\text { Disease target } \\
\text { (cholesterol), adherence } \\
\text { (chart review) }\end{array}$ & $\begin{array}{l}\text { High } \\
\text { risk of } \\
\text { bias }\end{array}$ \\
\hline $\begin{array}{l}\text { Goldstein, } \\
2005\end{array}$ & $\begin{array}{l}\text { Intervention on drug } \\
\text { choice for } \\
\text { hypertension }\end{array}$ & $\mathrm{CRCT}$ & $\begin{array}{l}\text { Family doctors, nurse } \\
\text { practitioners }\end{array}$ & $\begin{array}{l}\text { USA, multiple } \\
\text { sites }\end{array}$ & $\begin{array}{l}\text { Education, individual drug } \\
\text { profiles, follow up }\end{array}$ & Education & 9 months & $\begin{array}{l}\text { Education on } \\
\text { guidelines }\end{array}$ & $\begin{array}{l}\text { Disease target (BP), } \\
\text { adherence (prescription } \\
\text { and chart review) }\end{array}$ & $\begin{array}{l}\text { Low } \\
\text { risk of } \\
\text { bias }\end{array}$ \\
\hline Harris, 2005 & $\begin{array}{l}\text { Teleconferenced } \\
\text { educational detailing } \\
\text { for diabetes }\end{array}$ & $\mathrm{CRCT}$ & Family doctors & $\begin{array}{l}\text { Canada, } \\
\text { family } \\
\text { practices }\end{array}$ & $\begin{array}{l}\text { Eight one hour small group } \\
\text { educational sessions with } \\
\text { opinion leaders }\end{array}$ & Education & 3 months & $\begin{array}{l}\text { CME session } \\
\text { after } \\
\text { intervention } \\
\text { period }\end{array}$ & $\begin{array}{l}\text { Disease target (HbA1c), } \\
\text { adherence (chart } \\
\text { review) }\end{array}$ & $\begin{array}{l}\text { High } \\
\text { risk of } \\
\text { bias }\end{array}$ \\
\hline Hayes, 2002 & $\begin{array}{l}\text { Quality improvement } \\
\text { and written feedback } \\
\text { for CHF management }\end{array}$ & $\mathrm{CRCT}$ & $\begin{array}{l}\text { Hospitals, CHF } \\
\text { patients }\end{array}$ & USA, hospitals & $\begin{array}{l}\text { Education, quality } \\
\text { improvement tools from } \\
\text { liaisons, chart reminders }\end{array}$ & $\begin{array}{l}\text { Education + } \\
\text { audit and } \\
\text { feedback }\end{array}$ & 6 months & $\begin{array}{l}\text { Mailed quality } \\
\text { improvement } \\
\text { tools }\end{array}$ & $\begin{array}{l}\text { Disease target } \\
\text { (ventricular fxn), } \\
\text { adherence (chart } \\
\text { review) }\end{array}$ & $\begin{array}{l}\text { High } \\
\text { risk of } \\
\text { bias }\end{array}$ \\
\hline $\begin{array}{l}\text { Headrick, } \\
1992\end{array}$ & $\begin{array}{l}\text { Education and } \\
\text { feedback strategies to } \\
\text { improve compliance } \\
\text { with NCEP-PCEP } \\
\text { guidelines }\end{array}$ & $\mathrm{RCT}$ & Resident doctors & $\begin{array}{l}\text { USA. } \\
\text { Academic } \\
\text { hospital }\end{array}$ & $\begin{array}{l}\text { Lecture, chart reminders; } \\
\text { Lecture, patient specific } \\
\text { feedback and chart reminder }\end{array}$ & $\begin{array}{l}\text { Education + } \\
\text { Other } \\
\text { (reminders) }\end{array}$ & 20 weeks & Lecture alone & $\begin{array}{l}\text { Disease targets } \\
\text { (cholesterol), adherence } \\
\text { (chart review) }\end{array}$ & $\begin{array}{l}\text { Low } \\
\text { risk of } \\
\text { bias }\end{array}$ \\
\hline
\end{tabular}


Table 1 Characteristics of included studies (Continued)

\begin{tabular}{|c|c|c|c|c|c|c|c|c|c|c|}
\hline $\begin{array}{l}\text { Hendriks, } \\
2012\end{array}$ & $\begin{array}{l}\text { Nurse led guideline } \\
\text { based software } \\
\text { supported ICCP }\end{array}$ & RCT & $\begin{array}{l}\text { Family doctors, } \\
\text { specialists, patients } \\
\text { with atrial fibrillation }\end{array}$ & $\begin{array}{l}\text { Netherlands, } \\
\text { academic } \\
\text { center }\end{array}$ & $\begin{array}{l}\text { Nurse specialist educated } \\
\text { patients and CDSS }\end{array}$ & $\begin{array}{l}\text { Other (nurse } \\
\text { specialist) }\end{array}$ & 12 months & Usual care & $\begin{array}{l}\text { Mortality, } \\
\text { hospitalizations, quality } \\
\text { of life, adherence (chart } \\
\text { review) }\end{array}$ & $\begin{array}{l}\text { Low } \\
\text { risk of } \\
\text { bias }\end{array}$ \\
\hline $\begin{array}{l}\text { Kiessling, } \\
2011\end{array}$ & $\begin{array}{l}\text { Case based training to } \\
\text { optimize } \\
\text { hyperlipidemia care }\end{array}$ & $\mathrm{RCT}$ & $\begin{array}{l}\text { Family doctors, } \\
\text { patients with CHD }\end{array}$ & $\begin{array}{l}\text { Sweden, } \\
\text { primary } \\
\text { health care } \\
\text { centres }\end{array}$ & $\begin{array}{l}\text { Case based training seminars } \\
\text { and guideline provided }\end{array}$ & Education & 2 years & Usual care & $\begin{array}{l}\text { Mortality, disease target } \\
\text { (cholesterol), adherence } \\
\text { (prescription review) }\end{array}$ & $\begin{array}{l}\text { High } \\
\text { risk of } \\
\text { bias }\end{array}$ \\
\hline $\begin{array}{l}\text { Leonardis, } \\
2012\end{array}$ & $\begin{array}{l}\text { Multimodal } \\
\text { intervention to } \\
\text { improve adherence to } \\
\text { targets }\end{array}$ & cRCT & $\begin{array}{l}\text { Specialists, CKD } \\
\text { patients }\end{array}$ & $\begin{array}{l}\text { Italy, renal } \\
\text { clinics }\end{array}$ & $\begin{array}{l}\text { Education session, follow up } \\
\text { and audits }\end{array}$ & $\begin{array}{l}\text { Education + } \\
\text { audit and } \\
\text { feedback }\end{array}$ & 3 years & $\begin{array}{l}\text { Education and } \\
\text { standard care }\end{array}$ & $\begin{array}{l}\text { Mortality, } \\
\text { hospitalizations, quality } \\
\text { of life, disease target } \\
\text { (cholesterol), adherence } \\
\text { (prescription/ chart } \\
\text { review) }\end{array}$ & $\begin{array}{l}\text { Low } \\
\text { risk of } \\
\text { bias }\end{array}$ \\
\hline $\begin{array}{l}\text { Levine, } \\
2011\end{array}$ & $\begin{array}{l}\text { Multicomponent } \\
\text { internet delivered } \\
\text { intervention improve } \\
\text { CHD guideline } \\
\text { adherence }\end{array}$ & cRCT & $\begin{array}{l}\text { Family doctors, Ml } \\
\text { patients }\end{array}$ & $\begin{array}{l}\text { Virgin Islands } \\
\text { and Puerto } \\
\text { Rico, } \\
\text { community } \\
\text { primary care } \\
\text { clinics }\end{array}$ & $\begin{array}{l}\text { Educational cases, guidelines, } \\
\text { monthly update, reminders }\end{array}$ & $\begin{array}{l}\text { Education + } \\
\text { Other } \\
\text { (reminders) }\end{array}$ & 27 months & $\begin{array}{l}\text { Passive } \\
\text { dissemination }\end{array}$ & $\begin{array}{l}\text { Disease target } \\
\text { (cholesterol), adherence } \\
\text { (chart review) }\end{array}$ & $\begin{array}{l}\text { High } \\
\text { risk of } \\
\text { bias }\end{array}$ \\
\hline $\begin{array}{l}\text { Ornstein, } \\
2004\end{array}$ & $\begin{array}{l}\text { Multimethod quality } \\
\text { improvement } \\
\text { intervention for } \\
\text { adherence to quality } \\
\text { indicators in CVD and } \\
\text { stroke }\end{array}$ & CRCT & $\begin{array}{l}\text { Practice based } \\
\text { research network of } \\
\text { practices }\end{array}$ & $\begin{array}{l}\text { USA, primary } \\
\text { care practices }\end{array}$ & $\begin{array}{l}\text { Education, performance reports } \\
\text { quarterly, practice site visits and } \\
\text { network meetings (6-7 1-2 } \\
\text { day visits) with pharmacist } \\
\text { (academic detailing) }\end{array}$ & $\begin{array}{l}\text { Education }+ \\
\text { academic } \\
\text { detailing }\end{array}$ & 2 years & $\begin{array}{l}\text { Education, } \\
\text { performance } \\
\text { reports } \\
\text { quarterly }\end{array}$ & $\begin{array}{l}\text { Disease target (BP), } \\
\text { adherence (prescription, } \\
\text { chart review) }\end{array}$ & $\begin{array}{l}\text { High } \\
\text { risk of } \\
\text { bias }\end{array}$ \\
\hline $\begin{array}{l}\text { Petersen, } \\
2013\end{array}$ & $\begin{array}{l}\text { Effect of financial } \\
\text { incentives to reward } \\
\text { guideline based } \\
\text { hypertension care }\end{array}$ & CRCT & Family doctors & $\begin{array}{l}\text { USA, primary } \\
\text { care clinics }\end{array}$ & $\begin{array}{l}\text { Physician level incentives; } \\
\text { practice levels incetives; } \\
\text { combined (both) incentives }\end{array}$ & $\begin{array}{l}\text { Other } \\
\text { (incentives) }\end{array}$ & 20 months & Usual care & $\begin{array}{l}\text { Disease target (BP), } \\
\text { adherence (prescription, } \\
\text { chart review) }\end{array}$ & $\begin{array}{l}\text { High } \\
\text { risk of } \\
\text { bias }\end{array}$ \\
\hline $\begin{array}{l}\text { Peters- } \\
\text { Klimm, } \\
2009\end{array}$ & $\begin{array}{l}\text { Educational model for } \\
\text { GPs for the } \\
\text { management of CHF }\end{array}$ & CRCT & $\begin{array}{l}\text { Family doctors, CHF } \\
\text { patients }\end{array}$ & $\begin{array}{l}\text { Germany, } \\
\text { general } \\
\text { practitioner } \\
\text { clinics }\end{array}$ & $\begin{array}{l}\text { "Train the trainer" }= \\
\text { multidisciplinary andragogic } \\
\text { and didactic educational } \\
\text { sessions }\end{array}$ & $\begin{array}{l}\text { Education + } \\
\text { Other } \\
\text { (feedback) }\end{array}$ & 7 months & $\begin{array}{l}\text { Single } \\
\text { educational } \\
\text { session by } \\
\text { cardiologist }\end{array}$ & $\begin{array}{l}\text { Mortality, } \\
\text { hospitalizations, quality } \\
\text { of life, disease target } \\
\text { (course), adherence } \\
\text { (prescription review) }\end{array}$ & $\begin{array}{l}\text { Low } \\
\text { risk of } \\
\text { bias }\end{array}$ \\
\hline $\begin{array}{l}\text { Reutens, } \\
2012\end{array}$ & $\begin{array}{l}\text { Education of GPs on } \\
\text { the IDF-WPR guidelines } \\
\text { to improve metabolic } \\
\text { control }\end{array}$ & CRCT & $\begin{array}{l}\text { Family doctors, T2D } \\
\text { patients }\end{array}$ & $\begin{array}{l}\text { Asia-Pacfic, } \\
\text { general } \\
\text { practitioner } \\
\text { clinics }\end{array}$ & $\begin{array}{l}\text { Education meetings (two } \\
3 \text { months apart), reminder } \\
\text { letters and cards, flowsheet on } \\
\text { patient notes, patient diabetes } \\
\text { passport }\end{array}$ & $\begin{array}{l}\text { Education + } \\
\text { Other } \\
\text { (reminders, } \\
\text { diabetes } \\
\text { passport) }\end{array}$ & 12 months & $\begin{array}{l}\text { Instructed on } \\
\text { assessments in } \\
\text { study but no } \\
\text { information on } \\
\text { guidelines }\end{array}$ & $\begin{array}{l}\text { Disease target (BP), } \\
\text { adherence (chart } \\
\text { review) }\end{array}$ & $\begin{array}{l}\text { High } \\
\text { risk of } \\
\text { bias }\end{array}$ \\
\hline Rood, 2005 & $\begin{array}{l}\text { Computer based } \\
\text { guidelines to improve } \\
\text { nurse measurement of } \\
\text { patient glucose }\end{array}$ & RCT & ICU patients & $\begin{array}{l}\text { The } \\
\text { Netherlands, } \\
\text { teaching } \\
\text { hospital }\end{array}$ & $\begin{array}{l}\text { Guideline based advice via } \\
\text { computer decision support } \\
\text { software }\end{array}$ & $\begin{array}{l}\text { Other (decision } \\
\text { support tool) }\end{array}$ & 10 weeks & $\begin{array}{l}\text { Paper based } \\
\text { guideline } \\
\text { flowchart }\end{array}$ & $\begin{array}{l}\text { Disease target (glucose), } \\
\text { adherence (chart } \\
\text { review) }\end{array}$ & $\begin{array}{l}\text { High } \\
\text { risk of } \\
\text { bias }\end{array}$ \\
\hline
\end{tabular}


Table 1 Characteristics of included studies (Continued)

\begin{tabular}{|c|c|c|c|c|c|c|c|c|c|c|}
\hline Rossi, 1997 & $\begin{array}{l}\text { Guideline reminders to } \\
\text { improve prescribing } \\
\text { based on JNC V } \\
\text { guideline }\end{array}$ & CRCT & $\begin{array}{l}\text { Nurse practitioners, } \\
\text { hypertension patients }\end{array}$ & $\begin{array}{l}\text { USA, GIM } \\
\text { clinic }\end{array}$ & $\begin{array}{l}\text { Guideline reminder for } \\
\text { prescription and alternatives }\end{array}$ & $\begin{array}{l}\text { Other } \\
\text { (reminder) }\end{array}$ & 5 months & Usual care & $\begin{array}{l}\text { Disease target (BP), } \\
\text { adherence (prescription } \\
\text { review) }\end{array}$ & $\begin{array}{l}\text { High } \\
\text { risk of } \\
\text { bias }\end{array}$ \\
\hline $\begin{array}{l}\text { Roumie, } \\
2006\end{array}$ & $\begin{array}{l}\text { Multifactorial } \\
\text { intervention to } \\
\text { improve quality of } \\
\text { care of hypertension } \\
\text { patients }\end{array}$ & CRCT & $\begin{array}{l}\text { Physicians and nurse } \\
\text { practitioners, } \\
\text { hypertension patients }\end{array}$ & $\begin{array}{l}\text { USA, } \\
\text { community } \\
\text { and hospital } \\
\text { clinics }\end{array}$ & $\begin{array}{l}\text { Alert on medical record; } \\
\text { Educational sessions and alert } \\
\text { on medical record }\end{array}$ & $\begin{array}{l}\text { Education }+ \\
\text { other (alerts) }\end{array}$ & 6 months & $\begin{array}{l}\text { Providers } \\
\text { received email } \\
\text { with guideline }\end{array}$ & $\begin{array}{l}\text { Mortality, } \\
\text { hospitalizations, disease } \\
\text { target (BP), adherence } \\
\text { (prescription review) }\end{array}$ & $\begin{array}{l}\text { High } \\
\text { risk of } \\
\text { bias }\end{array}$ \\
\hline $\begin{array}{l}\text { Simon, } \\
2005\end{array}$ & $\begin{array}{l}\text { Academic detailing } \\
\text { individually or group } \\
\text { to increase diuretic } \\
\text { use in hypertension } \\
\text { patients }\end{array}$ & CRCT & $\begin{array}{l}\text { Family doctors, } \\
\text { hypertension patients }\end{array}$ & $\begin{array}{l}\text { USA, } \\
\text { community } \\
\text { health plan }\end{array}$ & $\begin{array}{l}\text { Academic detailing meeting } \\
\text { one-on-one; small group } \\
\text { academic detailing session }\end{array}$ & $\begin{array}{l}\text { Academic } \\
\text { detailing }\end{array}$ & 3 months & $\begin{array}{l}\text { Passive } \\
\text { dissemination }\end{array}$ & $\begin{array}{l}\text { Hospitalizations, disease } \\
\text { target(BP), adherence } \\
\text { (chart review) }\end{array}$ & $\begin{array}{l}\text { High } \\
\text { risk of } \\
\text { bias }\end{array}$ \\
\hline Steyn, 2013 & $\begin{array}{l}\text { Structured clinical } \\
\text { record and training } \\
\text { health care providers } \\
\text { to control diabetes } \\
\text { and hypertension }\end{array}$ & CRCT & $\begin{array}{l}\text { Nurses, patients with } \\
\text { diabetes and } \\
\text { hypertension }\end{array}$ & $\begin{array}{l}\text { South Africa, } \\
\text { community } \\
\text { health centres }\end{array}$ & $\begin{array}{l}\text { Structured record with } \\
\text { guideline embedded added } \\
\text { to patient folders, educational } \\
\text { package }\end{array}$ & Education & 1 year & $\begin{array}{l}\text { Passive } \\
\text { dissemination }\end{array}$ & $\begin{array}{l}\text { Disease target (HbA1c), } \\
\text { adherence (chart } \\
\text { review) }\end{array}$ & $\begin{array}{l}\text { High } \\
\text { risk of } \\
\text { bias }\end{array}$ \\
\hline $\begin{array}{l}\text { Svetkey, } \\
2009\end{array}$ & $\begin{array}{l}\text { Intervention to } \\
\text { increase physician } \\
\text { adherence to BP } \\
\text { guideline }\end{array}$ & CRCT & $\begin{array}{l}\text { Physicians, } \\
\text { hypertension patients }\end{array}$ & $\begin{array}{l}\text { USA, } \\
\text { community } \\
\text { practice }\end{array}$ & $\begin{array}{l}\text { CME courses, treatment } \\
\text { algorithm, quarterly feedback } \\
\text { on adherence }\end{array}$ & $\begin{array}{l}\text { Education }+ \\
\text { CME session + } \\
\text { other } \\
\text { (feedback) }\end{array}$ & 18 months & Usual care & $\begin{array}{l}\text { Disease target (BP), } \\
\text { adherence (chart } \\
\text { review) }\end{array}$ & $\begin{array}{l}\text { Low } \\
\text { risk of } \\
\text { bias }\end{array}$ \\
\hline $\begin{array}{l}\text { Tierney, } \\
2003\end{array}$ & $\begin{array}{l}\text { Decision support } \\
\text { system with guideline } \\
\text { for managing ischemic } \\
\text { heart disease and CHF } \\
\text { patients }\end{array}$ & RCT & $\begin{array}{l}\text { Pharmacists, CHF } \\
\text { patients }\end{array}$ & $\begin{array}{l}\text { USA, } \\
\text { academic } \\
\text { primary care } \\
\text { practice }\end{array}$ & $\begin{array}{l}\text { Physicians received patient } \\
\text { specific feedback; pharmacist } \\
\text { system to send feedback to } \\
\text { physicians; both }\end{array}$ & $\begin{array}{l}\text { Education }+ \\
\text { audit and } \\
\text { feedback }+ \\
\text { other (decision } \\
\text { support } \\
\text { system) }\end{array}$ & 1 year & Usual care & $\begin{array}{l}\text { Mortality, } \\
\text { hospitalizations, quality } \\
\text { of life, adherence (chart } \\
\text { review) }\end{array}$ & $\begin{array}{l}\text { High } \\
\text { risk of } \\
\text { bias }\end{array}$ \\
\hline $\begin{array}{l}\text { Van } \\
\text { Bruggen, } \\
2008\end{array}$ & $\begin{array}{l}\text { Facilitator enhanced } \\
\text { multifaceted } \\
\text { intervention for T2D } \\
\text { guideline } \\
\text { implementation }\end{array}$ & CRCT & $\begin{array}{l}\text { Family doctors and } \\
\text { nurses and practice } \\
\text { assistants, T2D } \\
\text { patients }\end{array}$ & $\begin{array}{l}\text { The } \\
\text { Netherlands, } \\
\text { primary care } \\
\text { practices }\end{array}$ & $\begin{array}{l}\text { Facilitators visited twice a } \\
\text { month to train staff on } \\
\text { guidelines, performance } \\
\text { feedback, }\end{array}$ & $\begin{array}{l}\text { Education + } \\
\text { audit and } \\
\text { feedback }\end{array}$ & 1 year & Usual care & $\begin{array}{l}\text { Disease target (HbA1c), } \\
\text { adherence (prescription } \\
\text { and chart review) }\end{array}$ & $\begin{array}{l}\text { Low } \\
\text { risk of } \\
\text { bias }\end{array}$ \\
\hline $\begin{array}{l}\text { Van } \\
\text { Steenkiste, } \\
2007\end{array}$ & $\begin{array}{l}\text { Decision support tool } \\
\text { for risk management } \\
\text { improving CVD } \\
\text { guideline performance }\end{array}$ & CRCT & $\begin{array}{l}\text { Family doctors, } \\
\text { patients without CVD }\end{array}$ & $\begin{array}{l}\text { The } \\
\text { Netherlands, } \\
\text { hospital }\end{array}$ & $\begin{array}{l}\text { Education, decision support } \\
\text { tool, }\end{array}$ & $\begin{array}{l}\text { Other (decision } \\
\text { support tool) }\end{array}$ & 8 months & $\begin{array}{l}\text { Educational } \\
\text { materials on } \\
\text { guideline }\end{array}$ & $\begin{array}{l}\text { Disease target (lifestyle), } \\
\text { adherence (chart } \\
\text { review) }\end{array}$ & $\begin{array}{l}\text { High } \\
\text { risk of } \\
\text { bias }\end{array}$ \\
\hline $\begin{array}{l}\text { Verweij, } \\
2013\end{array}$ & $\begin{array}{l}\text { Effectiveness of } \\
\text { guideline based care } \\
\text { on weight, CVD risk }\end{array}$ & CRCT & $\begin{array}{l}\text { Occupational } \\
\text { physicians }\end{array}$ & $\begin{array}{l}\text { The } \\
\text { Netherlands, } \\
\text { occupational } \\
\text { medicine }\end{array}$ & $\begin{array}{l}\text { Environment scan, patient } \\
\text { counselling training, patient } \\
\text { toolkit }\end{array}$ & $\begin{array}{l}\text { Other } \\
\text { (environment } \\
\text { scan, toolkit) }\end{array}$ & 18 months & Usual care & $\begin{array}{l}\text { Quality of life, disease } \\
\text { target (BP), adherence } \\
\text { (chart review) }\end{array}$ & $\begin{array}{l}\text { High } \\
\text { risk of } \\
\text { bias }\end{array}$ \\
\hline
\end{tabular}

Footnote: ${ }^{2}$ Risk of bias rated as high or low risk of bias based on overall domains, where high risk of bias designated if greater than 3 domains rated as high risk of bias 


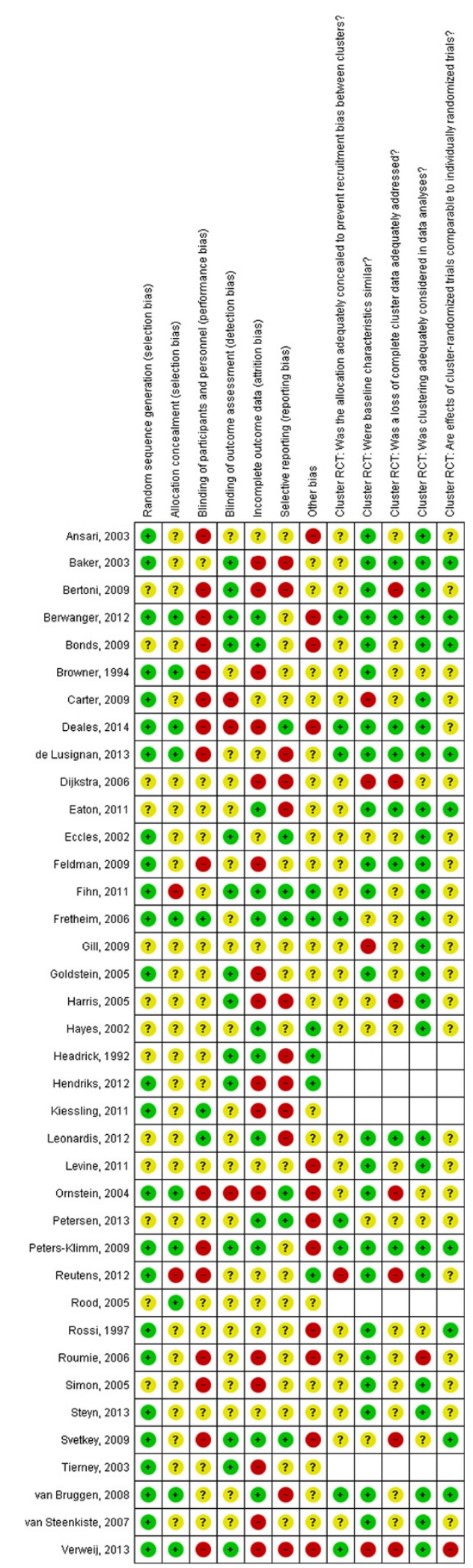

Fig. 2 Risk of bias summary table for each study. Green indicates a low risk of bias, yellow indicates unclear risk of bias and red indicates high risk of bias, as assessed by reviewers using the Cochrane risk of bias tool risk of bias studies on the overall estimate was possible for the meta-analysis of the effect of education on short term adherence outcomes. The pooled odds ratio was 2.36 (95\% CI 0.86 to 6.51) before studies with high risk of bias were excluded, and 3.65 (95\% CI 0.53 to 25.15 ) after studies with high risk of bias were excluded.

We compared results in studies that targeted physicians only in their intervention to interventions that involved non-physician healthcare providers alone or in addition to physicians with subgroup analysis. This subgroup analysis was possible in seven comparisons, and the subgroups of physician participants alone frequently had less heterogeneity than when grouped with all studies, suggesting participants may be a source of heterogeneity (Additional file 2: Figure S1). Another subgroup analysis we conducted compared results in studies that focused on an acute cardiovascular condition to a chronic cardiovascular condition. Five comparisons showed inconsistent results although the heterogeneity was reduced in at least one of the two subgroups in all comparisons.

\section{GRADE summary of findings tables}

The overall quality of evidence identified in this systematic review was moderate to very low due to high risk of bias, imprecision, and heterogeneity (Table 2). The most patient important outcome of mortality had moderate quality of evidence associated, indicating the results may be interpreted with some confidence.

\section{Discussion}

\section{Statement of principal findings}

We have focused on interventions aimed at improving adherence to CVD guidelines. Overall studies are variable in their conclusions on whether the intervention was effective, though our quantitative analysis supports that interventions trend towards having an impact on adherence to guidelines and patient outcomes. One comparison of an education intervention for the adherence outcome was statistically significant, indicating this area of study deserves further consideration, as these interventions may help improve both adherence to guidelines, and more importantly, patient outcomes. Our results were robust where sensitivity analyses were possible. Subgroup analyses (participant and condition) reduced the statistical heterogeneity but there was inconsistency in the subgroup with the larger effect for each analysis. In some cases, the physician subgroup favoured the intervention to a greater degree than the non-physician subgroup, but in other comparisons the opposite was true. The same results were found for the condition subgroup (acute vs. chronic condition). The confidence in these recommendations ranged from moderate to very low based on a GRADE summary of findings due to imprecision, risk of bias, and inconsistency. 


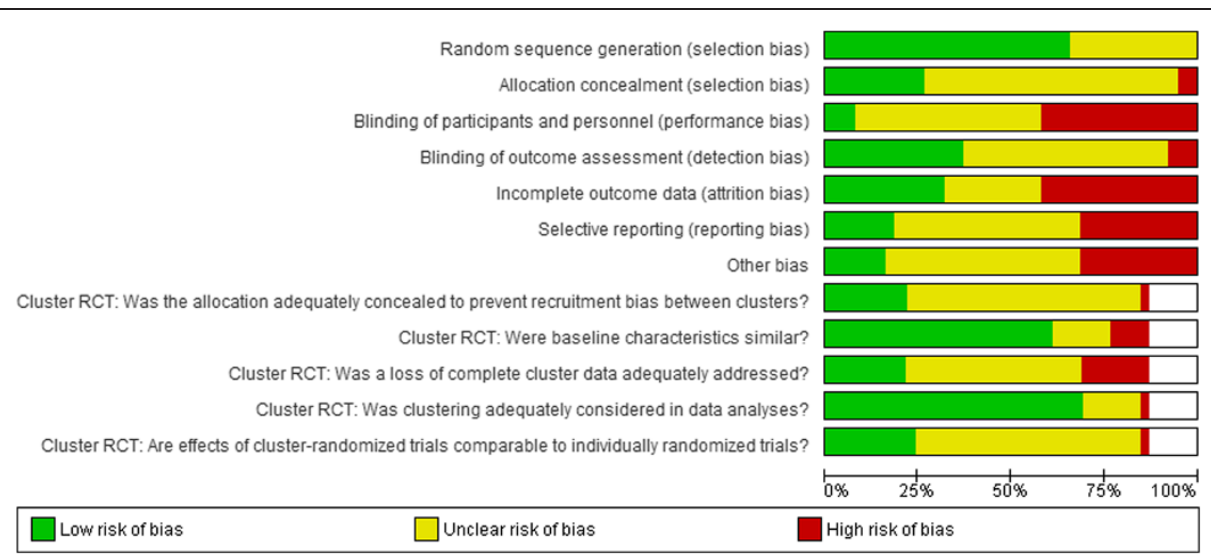

Fig. 3 Risk of bias summary graph, summarized for each domain. Green indicates a low risk of bias, yellow indicates unclear risk of bias and red indicates high risk of bias, as assessed by reviewers using the Cochrane risk of bias tool

\section{Strengths and weaknesses of the review}

Our systematic review has several strengths, including that it was comprehensive in inclusion of studies. We included all types of healthcare providers in order to illustrate the impact these interventions can have on both physicians and non-physicians, which is increasingly important for multidisciplinary teams required for complex diseases such as CVD. We limited our study inclusion to those that reported both adherence and a patient outcome, as interventions must improve both in order to be clinically useful. All screening, data extraction, and risk of bias assessment was done in duplicate with trained reviewers to ensure the reproducibility of these results. Our quantitative analysis was pre-specified to avoid finding spurious results due to post hoc analyses. We minimized the number of comparisons that were made while ensuring comparisons had fairly good clinical homogeneity to maintain the strength of those conclusions. We also contacted authors for missing data and to verify the accuracy of our data extractions of their trial, thus we have confidence in this data.

However, this review has limitations. The first relates to the quality of reporting in trials. Reporting of risk of bias domains was poor in many trials, making it difficult to assess risk of bias. There was also significant heterogeneity in the studies' interventions and characteristics making combining results in a meta-analysis difficult, leading to small numbers of studies included in each comparison. Meta-analyzing results was further complicated by uncertainty of the exact nature of some interventions due to limited descriptions of interventions available in publications. This also limited our

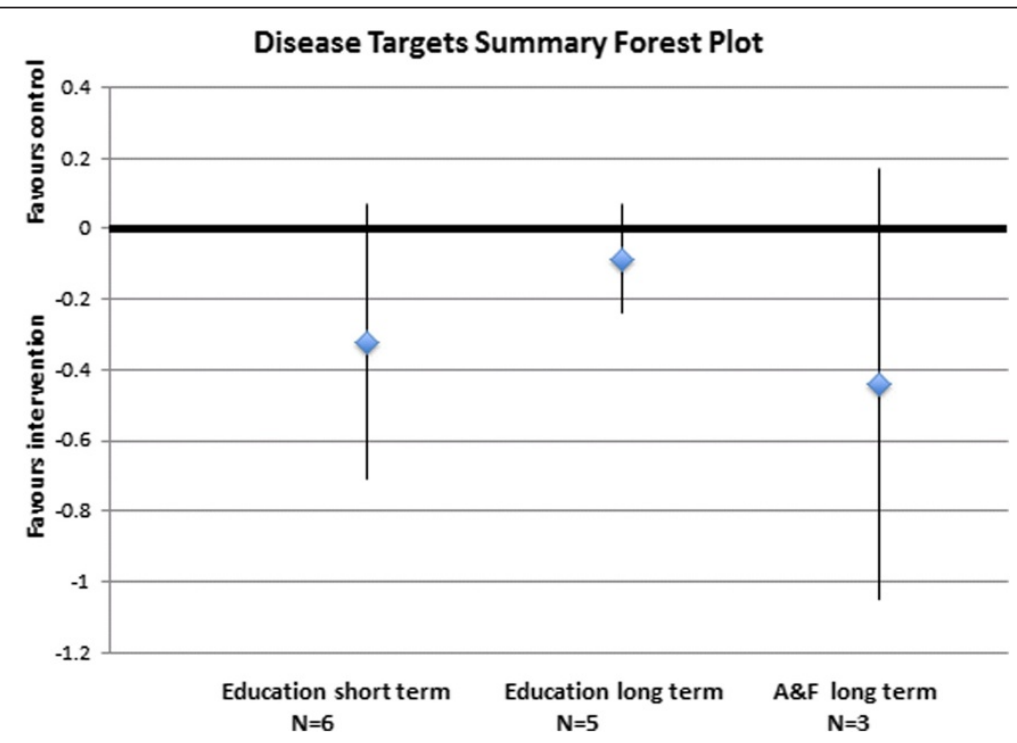

Fig. 4 Forest plot of education intervention comparison for continuous adherence outcome at a short term time point ( $<6$ months) 


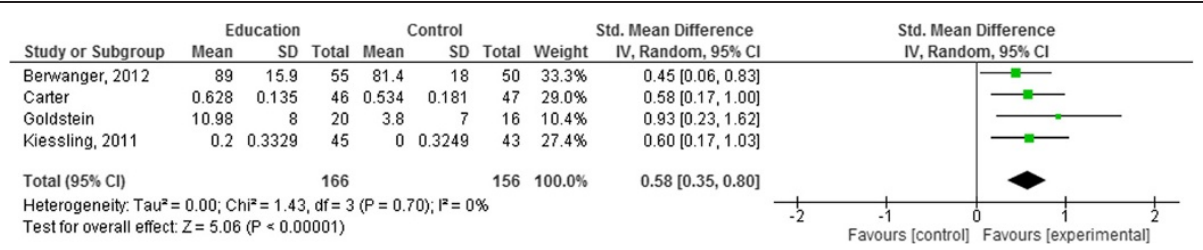

Fig. 5 Summary disease target outcome forest plot for three comparisons measured by standardized mean difference, with point estimate and $95 \% \mathrm{Cls}$

ability to assess publication bias, so we were unable to determine the effect that might have on our confidence in these results.

\section{Comparison to similar reviews}

A systematic review on CVD guideline implementation strategies in primary care physicians by Unverzagt et al. reported similar conclusions on the effectiveness of education and reminder system interventions to improve adherence [9]. Our review extends these findings, illustrating the impact at the patient level on mortality, hospitalizations, quality of life and disease targets, and to different healthcare providers.

Similar to our findings, a review by Grimshaw et al. on guideline implementation noted overall the most effective interventions tend to include specific educational interventions and patient specific reminders at point of care [6].

\section{Meaning of the review results}

These results indicate there is some evidence to support the use of some interventions to improve healthcare provider adherence to CVD guidelines. Despite the limitations in the studies in this review, a trend of interventions improving adherence and patient outcomes was noted, supporting that these interventions may be more effective than passive guideline dissemination strategies. However, more studies are needed to strengthen these conclusions.

The majority of interventions included were multifaceted, which some reviews have suggested provide positive outcomes more frequently than single interventions [53-55]. However, our results were not consistent with these; we found these interventions have limited effects, which may be related to the number of components in a given intervention, as only two interventions included all of the types of interventions. A review by Squires et al. found there is ambiguity in the evidence of whether multifaceted interventions are more effective than single interventions, which is in agreement with our inconsistent findings [55].

Another possible reason for the overall small effect sizes may relate to the complexity of the management of CVD. This includes treating and preventing multiple

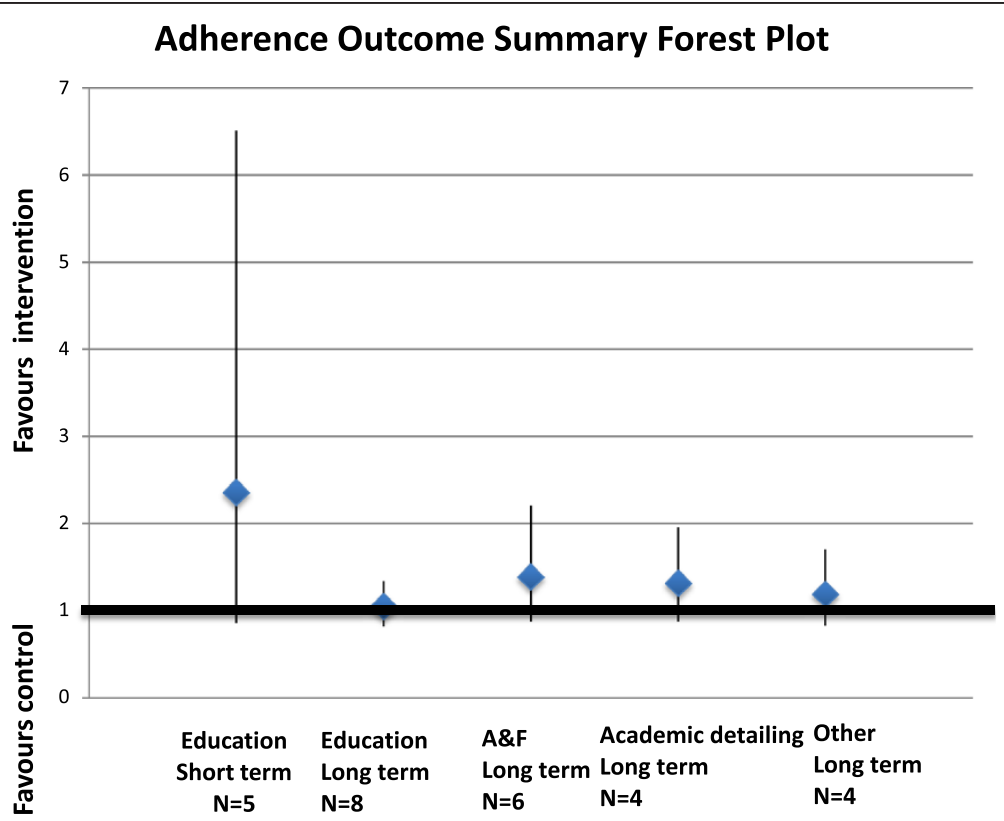

Fig. 6 Summary adherence outcome forest plot for five comparisons measured by odds ratio, with point estimates and $95 \% \mathrm{Cls}$ 
Table 2 Summary of findings table for educational interventions

\begin{tabular}{|c|c|c|c|c|c|c|}
\hline \multicolumn{7}{|c|}{$\begin{array}{l}\text { Education compared to control for improving adherence to cardiovascular disease guidelines } \\
\text { Patient or population: patients with improving adherence to cardiovascular disease guidelines } \\
\text { Settings: } \\
\text { Intervention: Education } \\
\text { Comparison: control }\end{array}$} \\
\hline \multirow[t]{2}{*}{ Outcomes } & \multicolumn{2}{|c|}{ Illustrative comparative risks ${ }^{\mathrm{a}}$ (95 \% Cl) } & \multirow{2}{*}{$\begin{array}{l}\text { Relative effect } \\
(95 \% \mathrm{Cl})\end{array}$} & \multirow{2}{*}{$\begin{array}{l}\text { No of } \\
\text { participants } \\
\text { (studies) }\end{array}$} & \multirow{2}{*}{$\begin{array}{l}\text { Quality of the } \\
\text { evidence } \\
\text { (GRADE) }\end{array}$} & \multirow[t]{2}{*}{ Comments } \\
\hline & Assumed risk control & Corresponding risk Education & & & & \\
\hline Mortality & Study population & & OR 0.54 & 2190 & $\oplus \oplus \oplus \Theta$ & \\
\hline \multirow[t]{5}{*}{ Follow-up: median 6 months } & 40 per 1000 & 22 per 1000 & (0.2 to 1.42$)$ & (3 studies) & moderate ${ }^{c}$ & \\
\hline & & (8 to 56$)$ & & & & \\
\hline & Moderate & & & & & \\
\hline & 26 per 1000 & 14 per 1000 & & & & \\
\hline & & $(5 \text { to } 37)^{\mathrm{b}}$ & & & & \\
\hline \multirow{3}{*}{$\begin{array}{l}\text { Disease Targets } \\
\text { Follow-up: } 3-6 \text { months }\end{array}$} & & The mean disease & & 2145 & $\oplus \ominus \Theta \Theta$ & SMD -0.32 \\
\hline & & $\begin{array}{l}\text { targets in the } \\
\text { intervention groups } \\
\text { was } 0.32 \text { standard } \\
\text { deviations lower }\end{array}$ & & (6 studies) & very low c,e,f & $(-0$. \\
\hline & & (0.71 lower to 0.07 higher) & & & & \\
\hline \multirow{3}{*}{$\begin{array}{l}\text { Adherence } \\
\text { Follow-up: 6-24 months }\end{array}$} & & The mean adherence & & 322 & $\oplus \oplus \oplus \oplus$ & SMD 0.58 \\
\hline & & $\begin{array}{l}\text { In the intervention } \\
\text { groups was } 0.58 \\
\text { standard deviations higher }\end{array}$ & & (4 studies) & high & \\
\hline & & (0.35 to 0.8 higher) & & & & \\
\hline Mortality & Study population & & OR 0.48 & 355 & $\oplus \oplus \ominus \ominus$ & \\
\hline \multirow[t]{5}{*}{ Follow-up: 7 months - 10 years } & 182 per 1000 & 96 per 1000 & (0.11 to 1.98$)$ & (4 studies) & $\operatorname{low}^{9}$ & \\
\hline & & (24 to 306$)$ & & & & \\
\hline & Moderate & & & & & \\
\hline & 146 per 1000 & 76 per 1000 & & & & \\
\hline & & $(18 \text { to } 253)^{b}$ & & & & \\
\hline Hospitalizations & Study population & & OR 0.88 & 979 & $\oplus \oplus \oplus \oplus$ & \\
\hline \multirow[t]{5}{*}{ Follow-up: 7-22 months } & 188 per 1000 & 170 per 1000 & (0.54 to 1.41$)$ & (4 studies) & high & \\
\hline & & (111 to 246$)$ & & & & \\
\hline & Moderate & & & & & \\
\hline & 191 per 1000 & 172 per 1000 & & & & \\
\hline & & $(113 \text { to } 250)^{b}$ & & & & \\
\hline Disease Targets & & The mean disease & & 2732 & $\oplus \oplus \ominus \ominus$ & SMD -0.09 \\
\hline \multirow[t]{2}{*}{ Follow-up: 7-27 months } & & $\begin{array}{l}\text { targets in the } \\
\text { intervention groups } \\
\text { was } 0.09 \text { standard } \\
\text { deviations lower }\end{array}$ & & (5 studies) & low $w^{f, h}$ & \\
\hline & & (0.24 lower to 0.07 higher) & & & & \\
\hline Adherence & Study population & & OR 1.05 & 6019 & $\oplus \oplus \ominus \ominus$ & \\
\hline \multirow[t]{5}{*}{ Follow-up: 7-27 months } & 609 per 1000 & 620 per 1000 & (0.82 to 1.34 ) & (8 studies) & low $w^{c, i}$ & \\
\hline & & (561 to 676$)$ & & & & \\
\hline & Moderate & & & & & \\
\hline & 236 per 1000 & 245 per 1000 & & & & \\
\hline & & $(202 \text { to } 293)^{b}$ & & & & \\
\hline Adherence & Study population & & OR 2.36 & 2145 & $\oplus \Theta \Theta \Theta$ & \\
\hline
\end{tabular}


Table 2 Summary of findings table for educational interventions (Continued)

\begin{tabular}{llll}
\hline Follow-up: median 6 months & 288 per 1000 & 489 per 1000 & (258 to 725$)$ \\
& Moderate & & \\
& 326 per 1000 & 533 per 1000 \\
& & $(294 \text { to } 759)^{\text {b }}$
\end{tabular}

${ }^{a}$ The basis for the assumed risk (e.g. the median control group risk across studies) is provided in footnotes. The corresponding risk (and its $95 \%$ confidence interval) is based on the assumed risk in the comparison group and the relative effect of the intervention (and its $95 \% \mathrm{Cl}$ )

Cl Confidence interval, OR Odds ratio

GRADE Working Group grades of evidence

High quality: Further research is very unlikely to change our confidence in the estimate of effect

Moderate quality: Further research is likely to have an important impact on our confidence in the estimate of effect and may change the estimate

Low quality: Further research is very likely to have an important impact on our confidence in the estimate of effect and is likely to change the estimate

Very low quality: We are very uncertain about the estimate

${ }^{\mathrm{b}}$ Assumed Risk is based on the default calculation within GRADEpro (mean control group risk, and median control group risk)

${ }^{\mathrm{C}}$ Assessment based on three studies thus precision cannot be accurately determined

${ }^{\mathrm{d}}$ Several included studies had 3 or more high risk of bias assessments

e Statistical heterogeneity $12=94 \%$

${ }^{f}$ Disease targets are an indirect estimate of patient important outcomes

${ }^{9}$ Statistical heterogeneity $12=70 \%$

${ }^{\text {h }}$ Statistical heterogeneity $12=41 \%$

'Statistical heterogeneity $12=60 \%$

jStatistical heterogeneity $12=95 \%$

${ }^{k}$ Overall estimate has large range for $95 \%$ confidence interval

risk factors in patients, such as diabetes, hypertension and dyslipidemia [56]. Most guidelines address only one of these diseases, and this may contribute to the small improvements found in this review. Given the multifactorial nature of CVD, it needs to be treated with guidelines that acknowledge this. Using harmonized CVD guidelines such as C-CHANGE is an important step that needs to be taken in CVD guideline implementation intervention trials to ensure the best, most comprehensive care is provided to patients [1]. This is also an important consideration as to why CVD guideline implementation strategies must differ from strategies used in treating simpler diseases such as pneumonia or asthma $[57,58]$.

\section{Unanswered questions and future research}

It would be beneficial for more high quality studies on this topic to be conducted to improve the strength of our recommendations, given the low confidence in most of these estimates due to a small number of studies included in each MA. Interventions should be fully described so they are not only reproducible, but future reviews are able to confidently determine homogeneous groups for meta-analyses. Future reviews on this topic should also define clinically important differences to determine whether the effects are not only statistically significant, but clinically significant as well.

\section{Conclusions}

Interventions to improve adherence to CVD guidelines can be effective at improving both adherence and patient outcomes, and are often more effective than guideline dissemination alone. Interventions that focused on healthcare provider education demonstrated statistically significant improvements. The overall quality of evidence available in this review was low, but several patient important outcomes including mortality were supported by moderate to high quality evidence.

\section{Additional files}

\section{Additional file 1: Supplementary: Medline search strategy.}

(DOCX $14 \mathrm{~kb}$ )

Additional file 2: Figure S1. Figure A: Subgroup analysis of physician participants compared to other participants in education focused intervention trials disease target outcome at a short term time point. (DOCX $30 \mathrm{~kb}$ )

\section{Abbreviations}

CVD: Cardiovascular disease; CPG: Clinical practice guideline; MA: Meta-analysis; RCT: Randomized controlled trials; GRADE: Grading of Recommendations Assessment, Development and Evaluation; CME: Continuing medical education; OR: Odds ratio; SMD: Standardized mean difference; Cl: Confidence interval.

\section{Competing Interests}

CVZ received a research grant through Boehringer-Ingelheim. All other authors declare no conflicts of interest.

\section{Authors' contributions}

RAJ conceived of the study and its design, coordinated and participated in data extraction and carried out statistical analyses, and drafted the manuscript. MJT participated in screening and data extraction. GHC participated in screening. $C T$ participated in screening. AC participated in data extraction. CVZ helped conceive of the study. JH helped plan the design and data analysis, and helped draft the manuscript. All authors read, revised, and approved the final manuscript.

\section{Authors' information}

RAJ, GHC and MJT are medical students at Dalhousie University. CT is an undergraduate science student at St. Mary's University. JH is an associate professor in Epidemiology at Dalhousie University and is head of the Nova Scotia Cochrane Resource Centre. CVZ is a general internist and an assistant professor in the Department of Medicine at Dalhousie University. 


\section{Availability of data and materials}

\author{
Not applicable.
}

\section{Acknowledgements}

We acknowledge the assistance of Robin Parker for advice during the creation of our search strategy. RAJ received funding from the DMRF Leo Alexander Summer Research Studentship. JH held funding from the Nova Scotia Health Research Foundation to support local systematic review activities. MJT and GHC received funding through the RIM summer research program. CVZ has received a research grant through Boehringer-Ingelheim. All funding bodies had no influence on the conception, design or interpretation of data.

\section{Funding}

RAJ received funding from the DMRF Leo Alexander Summer Research Studentship. JAH held funding from the Nova Scotia Health Research Foundation to support local systematic review activities. MJT and GHC received funding through the RIM summer research program. CVZ has received a research grant through Boehringer-Ingelheim. All funding bodies had no influence on the conception, design or interpretation of data

\section{Author details}

${ }^{1}$ Faculty of Medicine, Dalhousie University, Mailbox 354, 5849 University Avenue, Halifax, NS, CanadaB3H 4R2. 'Department of Science, St. Mary's University, Halifax, Canada. ${ }^{3}$ Department of Medicine, Dalhousie University, Halifax, Canada. ${ }^{4}$ Department of Community Health and Epidemiology, Dalhousie University, Halifax, Canada.

\section{Received: 1 May 2015 Accepted: 11 September 2015}

Published online: 22 October 2015

\section{References}

1. Tobe SW, Stone JA, Brouwers M, Bhattacharyya O, Walker KM, Dawes M, et al. Harmonization of guidelines for the prevention and treatment of cardiovascular disease: the C-CHANGE Initiative. Can Med Assoc J. 2011;183(15):e1135-50.

2. Perk J, De Backer G, Gohlke H, Graham I, Reiner Z, Verschuren WM, et al. European Guidelines on cardiovascular disease prevention in clinical practice (version 2012): Addenda The Fifth Joint Task Force of the European Society of Cardiology. Eur Heart J. 2012. doi:10.1093/eurheart/ehs165.

3. Indio do Brasil CKO, Avezum A, Uint L, Del Monaco MI, De Barros VM, Campos SYR, et al. Cardiovascular prevention in coronary heart disease patients: guidelines implementation in clinical practice. Rev Bras Cir Cardiovasc. 2013;28(2):238-47.

4. Ray KK, Kastelein JJP, Boekholdt SM, Nicholls SJ, Khaw KT, Ballantyne CM, et al. The ACC/AHA 2013 guideline on the treatment of blood cholesterol to reduce atherosclerotic cardiovascular disease risk in adults: The good the bad and the uncertain: A comparison with ESC/EAS guidelines for the management of dyslipidaemias 2011. Eur Heart J. 2014;35:960-8.

5. Stone NJ, Robinson JG, Lichtenstein AH, Merz CNB, Blum CB, Eckel RH, et al. ACC/AHA Guideline on the Treatment of Blood Cholesterol to Reduce Atherosclerotic Cardiovascular Risk in Adults A Report of the American College of Cardiology/American Heart Association Task Force on Practice Guidelines. Circulation. 2014;129 suppl 2:S1-S45.

6. Grimshaw JM, Russell IT. Effect of clinical guidelines on medical practice: a systematic review of rigorous evaluations. Lancet. 1993;342:1317-22.

7. Feifer C, Ornstein SM, Jenkins RG, Wessell A, Corley ST, Nemeth LS, et al. The logic behind a multimethod intervention to improve adherence to clinical practice guidelines in a nationwide network of primary care practices. Eval Health Prof. 2006:29:65-88

8. Davis DA, Taylor-Vaisey A. Translating guidelines into practice. Can Med Assoc J. 1997:157:408-16.

9. Unverzagt S, Oemler M, Braun K, Klement A. Strategies for quideline implementation in primary care focusing on patients with cardiovascular disease: a systematic review. Fam Pract. 2014;31(3):247-66.

10. Higgins J, Green S. Cochrane Handbook for Systematic Reviews of Interventions Version 5.1.0 [Updated March 2011]. The Cochrane Collaboration, 2011. Available from www.cochrane-handbook.org.

11. McKenzie J, Ryan R, Di Tanna GL; Cochrane Consumers and Communication Review Group. 'Cochrane Consumers and Communication Review Group: cluster randomised controlled trials'. http://cccrg.cochrane.org, March 2014 (December 20, 2014).
12. Ansari M, Shlipak MG, Heidenreich PA, Van Ostaeyen D, Pohl EC, Browner WS, et al. Improving guideline adherence: A randomized trial evaluating strategies to increase beta-blocker use in heart failure. Circulation. 2003:107:2799-804

13. Baker R, Fraser RC, Stone M, Lambert P, Stevenson K, Shiels C. Randomised controlled trial of the impact of guidelines, prioritised review criteria and feedback on implementation of recommendations for angina and asthma. Br J Gen Pract. 2003:53:284-91.

14. Bertoni AG, Bonds DE, Chen H, Hogan P, Crago L, Rosenberger E, et al. Impact of a multifaceted intervention on cholesterol management in primary care practices. Public Health. 2009;169(7):678-86.

15. Browner WS, Baron RB, Solkowitz S, Adler LJ, Gullion DS. Physician management of hypercholesterolemia. A randomized trial of continuing medical education. West J Med. 1994;161:572-8.

16. Carter BL, Ardery G, Dawson JD, James P, Bergus GR, Doucette WR, et al. Physician and pharmacist collaboration to improve blood pressure control. Arch Intern Med. 2009;169(21):1996-2002.

17. Deales A, Fratini M, Romano S, Rappelli A, Penco M, Perna GP, et al. Care manager to control cardiovascular risk factors in primary care: The Raffaello cluster randomized trial. Nutr Metab Cardiovasc Dis. 2014;24(5):563-71.

18. de Lusignan S, Gallagher H, Chan T, Thomas N, van Vlymen J, Nation M, et al. The QICKD study protocol: a cluster randomised trial to compare quality improvement interventions to lower systolic BP in chronic kidney disease (CKD) in primary care. Implement Sci. 2009;4:39-54.

19. Dijkstra RF, Niessen LW, Braspenning JCC, Adang E, Grol R. Patient-centred and professional-directed implementation strategies for diabetes guidelines: A cluster-randomized trial-based cost-effectiveness analysis. Diabet Med. 2006;23:164-70.

20. Eccles M, Hawthorne G, Whitty P, Steen N, Vanoli A, Grimshaw J, et al. A randomised controlled trial of a patient based Diabetes Recall and Management System: the DREAM trial: a study protocol [ISRCTN32042030]. BMC Health Serv Res. 2002;2(1):5.

21. Feldman RD, Zou GY, Vandervoort RK, Wong CJ, Nelson SE, Feagan BG. A simplified approach to the treatment of uncomplicated hypertension: a cluster randomized, controlled trial. Hypertension. 2009;53:646-53.

22. Fihn SD, Bucher JB, McDonell M, Diehr P, Rumsfeld JS, Doak M, et al. Collaborative Care Intervention for Stable Ischemic Heart Disease. Arch Intern Med. 2011;171(16):1471-9.

23. Fretheim A, Oxman AD, Håvelsrud K, Treweek S, Kristoffersen DT, Bjørndal A. Rational prescribing in primary care (RaPP): A cluster randomized trial of a tailored intervention. PLoS Med. 2006:3(6):0783-91.

24. Fretheim A, Oxman AD, Treweek S, Bjørndal A. Rational Prescribing in Primary Care (RaPP-trial). A randomised trial of a tailored intervention to improve prescribing of antihypertensive and cholesterol-lowering drugs in general practice [ISRCTN48751230]. BMC Health Serv Res. 2003;3:5-13.

25. Fretheim A, Oxman AD, Flottorp S. Improving prescribing of antihypertensive and cholesterol-lowering drugs: a method for identifying and addressing barriers to change. BMC Health Serv Res. 2004;4(1):23.

26. Gill JM, Chen YX, Glutting JJ, Diamond JJ, Lieberman MI. Impact of decision support in electronic medical records on lipid management in primary care. Popul Health Manag. 2009;12(5):221-6.

27. Harris SB, Leiter LA, Webster-Bogaert S, Van DM, O'Neill C. Teleconferenced educational detailing: diabetes education for primary care physicians. J Contin Educ Health Prof. 2005:25:87-97.

28. Hayes RP, Baker DW, Luthi JC, Baggett RL, McClellan W, Fitzgerald D, et al. The effect of external feedback on the management of medicare inpatients with congestive heart failure. Am J Med Qual. 2002;17:225-35.

29. Headrick LA, Speroff T, Pelecanos H, Cebul RD. Efforts to improve compliance with the national cholesterol education program guidelines. Arch Intern Med. 1992;152:2490-96.

30. Hendriks JML, De Wit R, Crijns H, Vrijhoef H, Prins M, Pisters R, et al. Nurseled care vs. usual care for patients with atrial fibrillation: Results of a randomized trial of integrated chronic care vs. routine clinical care in ambulatory patients with atrial fibrillation. Eur Heart J. 2012;33:2692-9.

31. Leonardis D, Mallamaci F, Enia G, Postorino M, Tripepi G, Zoccali C. The MAURO study: Baseline characteristics and compliance with guidelines targets. J Nephrol. 2012;25(5):1081-90.

32. Levine DA, Funkhouser EM, Houston TK, Gerald JK. Improving Care After Myocardial Infarction Using a 2-Year Internet-Delivered Intervention. Arch Intern Med. 2014;171(21):1910-7. 
33. Ornstein S, Jenkins RG, Nietert PJ, Feifer C, Roylance LF, Nemeth L. Improving Patient Care A Multimethod Quality Improvement Intervention To Improve Preventive Cardiovascular Care. Ann Intern Med. 2004;141:523-32.

34. Petersen LA, Simpson K, Pietz K, Urech TH, Hysong SJ, Profit J, et al. Effects of individual physician-level and practice-level financial incentives on hypertension care: a randomized trial. JAMA. 2013;310(10):1042-50.

35. Petersen LA, Urech L, Simpson K, Pietz K, Hysong SJ, Profit J, et al. Design, rationale, and baseline characteristics of a cluster randomized controlled trial of pay for performance for hypertension treatment: study protocol. Implement Sci. 2011;6(1):114.

36. Peters-Klimm F, Campbell S, Müller-Tasch T, Schellberg D, Gelbrich G, Herzog W, et al. Primary care-based multifaceted, interdisciplinary medical educational intervention for patients with systolic heart failure: lessons learned from a cluster randomised controlled trial. Trials. 2009;10:68.

37. Peters-Klimm F, Müller-Tasch T, Remppis A, Szecsenyi J, Schellberg D. Improved guideline adherence to pharmacotherapy of chronic systolic heart failure in general practice - Results from a cluster-randomized controlled trial of implementation of a clinical practice guideline. J Eval Clin Pract. 2008;14:823-9.

38. Reutens AT, Hutchinson R, Van Binh T, Cockram C, Deerochanawong C, Ho LT, et al. The GIANT study, a cluster-randomised controlled trial of efficacy of education of doctors about type 2 diabetes mellitus management guidelines in primary care practice. Diabetes Res Clin Pract. 2012;98(1):38-45.

39. Rood E, Bosman R, Van der Spoel J, Taylor P, Zandstra D. Use of a computerized guideline for glucose regulation in the ICU improved both guideline adherence and glucose regulation. J Am Med Inform Assoc. 2005:12:172-80.

40. Rossi RA, Every NR. A computerized intervention to decrease the use of calcium channel blockers in hypertension. J Gen Intern Med. 1997;12:672-8.

41. Simon SR, Majumdar SR, Prosser LA, Salem-Schatz S, Warner C, Kleinman K, et al. Group versus individual academic detailing to improve the use of antihypertensive medications in primary care: A cluster-randomized controlled trial. Am J Med. 2005;118:521-8.

42. Steyn K, Lombard C, Gwebushe N, Fourie JM, Everett-Murphy K, Zwarenstein $M$, et al. Implementation of national guidelines, incorporated within structured diabetes and hypertension records at primary level care in Cape Town, South Africa: A randomised controlled trial. Glob Health Action. 2013;6:1-9.

43. Svetkey LP, Pollak KI, Yancy WS, Dolor RJ, Batch BC, Samsa G, et al. Hypertension improvement project: Randomized trial of quality improvement for physicians and lifestyle modification for patients. Hypertension. 2009;54:1226-33.

44. Tierney WM, Overhage JM, Murray MD, Harris LE, Zhou X, Eckert GJ, et al. Effects of computerized guidelines for managing heart disease in primary care. J Gen Intern Med. 2003;18:967-76.

45. van Bruggen R, Gorter KJ, Stolk RP, Verhoeven RP, Rutten GE. Implementation of locally adapted guidelines on type 2 diabetes. Fam Pract. 2008;25:430-7.

46. van Steenkiste B, van der Weijden T, Stoffers HEJH, Kester ADM, Timmermans DRM, Grol R. Improving cardiovascular risk management: a randomized, controlled trial on the effect of a decision support tool for patients and physicians. Eur J Cardiovasc Prev Rehabil. 2007;14:44-50.

47. Verweij LM, Proper Kl, Weel AN, Hulshof CTJ, van Mechelen W. Long-term effects of an occupational health guideline on employees' body weightrelated outcomes, cardiovascular disease risk factors, and quality of life: results from a randomized controlled trial. Scand J Work Environ Health. 2013;39(3):284-94.

48. Verweij LM, Proper KI, Weel ANH, Hulshof CTJ, van Mechelen W. Design of the Balance@Work project: systematic development, evaluation and implementation of an occupational health guideline aimed at the prevention of weight gain among employees. BMC Public Health. 2009;9:461.

49. Kiessling A, Lewitt M, Hendriksson P. Case-based training of evidence-based clinical practice in primary care with decreased mortality in patients with coronary heart disease. Ann Fam Med. 2011;9:211-18.

50. Roumie C, Elasy T, Greevy R, Griffin M, Liu X, Stone W, et al. Improving Blood Pressure Control through Provider Education, Provider Alerts, and Patient Education. Ann Intern Med. 2006;145:165-75.

51. Bonds D, Hogen P, Bertoni A, Chen H, Clinch C, Hiott A, et al. A multifaceted intervention to improve blood pressure control: The Guideline Adherence for Health Health (GLAD) study. Am J Manag Care. 2010;157(2):278-84.
52. Eaton C, Parker D, Borkan J, McMurray J, Roberts M, Lu B, et al. Translating Cholesterol Guidelines Into Primary Care Practice: A Multimodal Cluster Randomized Trial. Ann Fam Med. 2011;9:528-37.

53. Berwanger O, Guimaraes H, Larangeira L, Cavalcanti A, Kodama A, Zazula A, et al. Effect of a multifaceted intervention on use of evidence-based therapies in patients with acute coronary syndromes in Brazil: The BRIDGEACS randomized trial. JAMA. 2012;307(19):2041-49.

54. Goldstein M, Lavori P, Coleman R, Advani A, Hoffman B. Improving adherence to guidelines for hypertension drug prescribing: clusterrandomized controlled trial of general versus patient-specific recommendations. Am J Manag Care. 2005;11:677-85.

55. Squires JE, Sullivan K, Eccles MP, Worswick J, Grimshaw JM. Are multifaceted interventions more effective than single-component interventions in changing health-care professionals' behaviours? An overview of systematic reviews. Implement Sci. 2014;9:152.

56. Reiner Z, Sonicki Z, Tedeschi-Reiner R. Physicians perception, knowledge and awareness of cardiovascular risk factors and adherence to prevention guidelines: The PERCRO-DOC survey. Atherosclerosis. 2010;213:598-603.

57. Okelo SO, Butz AM, Sharma R, Diette GB, Pitts SI, King TM, et al. Interventions to modify health care provider adherence to asthma guidelines. Rockville (MD): AHRQ Publication. 2013;95.

58. Ambroggio L, Thomson J, Murtagh Kurowski E, Courter J, Statile A, Graham C, et al. Quality improvement methods increase appropriate antibiotic prescribing for childhood pneumonia. Pediatrics. 2013;131:e1623-31.

\section{Submit your next manuscript to BioMed Central and take full advantage of:}

- Convenient online submission

- Thorough peer review

- No space constraints or color figure charges

- Immediate publication on acceptance

- Inclusion in PubMed, CAS, Scopus and Google Scholar

- Research which is freely available for redistribution 